\title{
Mathematical model of non-basal testosterone regulation in the male by pulse modulated feedback
}

\author{
Alexander Churilov ${ }^{\mathrm{a}}$ Alexander Medvedev ${ }^{\mathrm{b}, 1}$ \\ Alexander Shepeljavyi ${ }^{\text {a }}$ \\ ${ }^{a}$ Faculty of Mathematics and Mechanics, St. Petersburg State University, Universitetsky \\ av. 28, Peterhof, 198504, St. Petersburg, Russia \\ ${ }^{\mathrm{b}}$ Information Technology, Uppsala University, SE-751 05 Uppsala, Sweden
}

\begin{abstract}
A parsimonious mathematical model of pulse modulated regulation of non-basal testosterone secretion in the male is developed. The model is of third differential order, reflecting the three most significant hormones in the regulation loop, but is yet shown to be capable of sustaining periodic solutions with one or two pulses of gonadotropin-releasing hormone $(\mathrm{GnRH})$ on each period. Lack of stable periodic solutions is otherwise a main shortcoming of existing low-order hormone regulation models. Existence and stability of periodic solutions are studied. The periodic mode with two GnRH pulses on the least period has not been described in medical literature but is found to explain experimental data well.
\end{abstract}

Key words: Biomedical systems, impulse signals, oscillation, limit cycles

\section{Introduction}

Hormonal (endocrine) regulation is a complex biological system where hormones, often measured as their serum concentrations, interact via numerous feedback and feed-forward relationships, see [17,7]. Regarding mathematical modeling of hormone dynamics, one considers two general tendencies - hormone clearing from the blood which implies decrease of the serum concentration and hormone secretion contributing new amounts of the hormone into the blood stream. Clearing rate is basically proportional to the hormone concentration while secretion of a hormone is defined by concentration and dynamics of other hormones. Concentration rise in a hormone can either stimulate secretion of a given hormone or inhibit it. In this way,

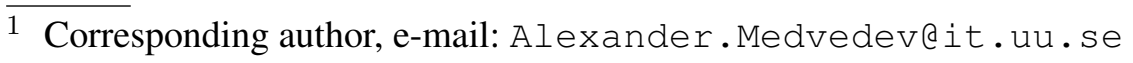


positive and negative feedbacks arise between different hormone concentrations in the blood stream of an organism. The loop of interacting hormones is closed and dynamically stable which guarantees homeostasis, i.e. biological self-regulation. To correct the dynamic behaviors of endocrine systems, exogenous signals can be used, e.g. medication, different kinds of medical treatment, physical activity, special diet, etc.

In the endocrine system of testosterone ( $\mathrm{Te}$ ) regulation in the male, essential role is played by the luteinizing hormone (LH) and gonadotropin-releasing hormone $(\mathrm{GnRH})$. While Te is produced in testes, $\mathrm{LH}$ and $\mathrm{GnRH}$ are secreted in different parts of the brain - hypophysis (pituitary gland) and hypothalamus, respectively. Therefore, the dynamics of $\mathrm{LH}$ and GnRH are closely related to the neural dynamics. The secretion of GnRH stimulates the secretion of LH which, in its turn, stimulates the production of Te, while Te inhibits the secretion of GnRH and $\mathrm{LH}$ [24].

Experimental studies based on high-resolution time assay series reveal (see e.g. [21]) that concentrations of Te and $\mathrm{LH}$ in the adult male exhibit oscillative behavior and their exact signal form depends on the individual. Direct measurements of GnRH in the human are difficult to implement due to ethical reasons but experiments on animals confirm that also secretion of $\mathrm{GnRH}$ is oscillative and it is furthermore pulsatile, see e.g. [15]. Oscillations in hormone concentrations are of a broad spectrum. Ultraradian harmonics with a period of $1-3$ hours, depending on the individual, are present as well as circadian rhythm of 24 hours is clearly observed. Children who do not reached puberty and adults suffering from e.g. catatonic schizophrenia do not have significant variation in the hormone levels, [21].

In this paper, periodic solutions of a recently suggested in [2] low-order mathematical model of the GnRH-LH-Te axis are studied. The model takes into account the pusatile nature of GnRH release and is shown to be capable of sustained oscillations of different types. The novelty of the model is that it combines relative simplicity facilitating stringent mathematical analysis with sufficient flexibility allowing for its use in parameter estimation.

First, a brief overview of results related to mathematical modeling of testosterone regulation in the male is provided to highlight the gap filled in by the model in hand. Then existence and stability results for periodic solutions of the model are presented. The model analysis suggests that periodic solutions with one or two $\mathrm{GnRH}$ pulses are feasible. It is proven that a periodic solution with one pulse of $\mathrm{GnRH}$ is unique for a given set of model parameters. In the periodic mode with two $\mathrm{GnRH}$ pulses, the model is found to be consistent with biological data. 


\section{Mathematical models of testosterone regulation}

Secretion of the hormones comprises two components - basal and pulsatile (nonbasal). Basal secretion of Te is a slow continuous process while the pulsatile one is directed by episodic release of GnRH.

A mathematically sensible model of the GnRH-LH-Te axis has first been suggested in [21]. It comprises three differential equations

$$
\begin{aligned}
\dot{R} & =f(T)-B_{1}(R), \\
\dot{L} & =G_{1}(R)-B_{2}(L), \\
\dot{T} & =G_{2}(L)-B_{3}(T),
\end{aligned}
$$

where $R(t), L(t)$ and $T(t)$ represent the serum concentrations of $\mathrm{GnRH}$ (termed as LHRH in [21]), LH and Te, respectively. The pulsatile nature of GnRH secretion, already discovered at that time [4], is not taken into account in (1).

Functions $B_{1}, B_{2}, B_{3}$ describe clearing rates of the hormones and $G_{1}, G_{2}, f$ are the rates of their secretion. All those are non-negative quantities. The first five functions are non-decreasing and can approximately be regarded as linear ones

$$
\begin{aligned}
B_{i}(x) & =b_{i} x, \quad i=1,2,3, \\
G_{i}(x) & =g_{i} x, \quad i=1,2,
\end{aligned}
$$

where $b_{i}, g_{i}$ are positive numbers. Therefore, (1) takes the form

$$
\begin{aligned}
\dot{R} & =f(T)-b_{1} R, \\
\dot{L} & =g_{1} R-b_{2} L, \\
\dot{T} & =g_{2} L-b_{3} T .
\end{aligned}
$$

As pointed out in [1], the linearity assumption is an idealization. In practice, these functions go into saturation for large values of the argument. Function $f(x)$ is nonincreasing and highly nonlinear. In [21], analytical sufficient conditions for (2) to have a stable periodic solution are given. Specialized forms of $f(x)$ are studied in more detail. If $f(x)$ is chosen to be a Hill function [7], $f(x)=K /\left(1+\beta x^{\rho}\right)$ (where all parameters are positive), then the condition for the existence of a periodic condition is $\rho>8$. According to $[17,13]$ this value of $\rho$ is not realistic. In fact, it is not unusual that extremely high values of $\rho$ (up to several hundreds, see [18]) are necessary to achieve limit cycles in the system. Another possible choice is a nonlinearity involving Heaviside function (unit step). The resulting system of equations has been studied by simulation in [18].

In [22], model (1) has been extended to account for the delay in the transport of LH from hypophysis to testes. Besides, a periodic excitation describing circadian rhythm has been introduced. 
The models by Smith have been further studied and followed up on by other researchers $[1,17,6,5]$.

The Smith models and their modifications are illustrative and give an idea about the general tendencies of the considered endocrine system. Unfortunately, they poorly correspond to the experimentally observed behaviors that are far from being periodic and also include irregular components.

Existing stochastic models demonstrate, as a rule, better agreement with clinical data compared to the models with regular dynamics. The most complete stochastic model of the GnRH-LH-Te axis, taking into account quite subtle biological considerations, is suggested in $[26,14]$. The model has been developed on the basis of $\mathrm{LH}$ and Te assays taken from a large number of patients with sampling time of $10 \mathrm{~min}$. The concentrations of GnRH, LH and Te are described by the system of stochastic differential equations. Modifications of the model that take into account circadian rhythm and external disturbances can also be found in [26,14]. Clearly, the stochastic model $[26,14]$ is quite complex and involves a significant number of adjustable parameters. This makes it possible to coerce the model into many different kinds of dynamical behavior but, at the same time, makes it the best of the currently available ones at explaining experimental data.

A quite simple stochastic model based on handling random events was suggested in [13].

\section{Pulse modulation model of testosterone regulation}

As follows from the preceding sections, there is no single broadly accepted mathematical model of testosterone regulation in the male. Furthermore, the biological mechanism of the involved feedbacks is not at all clear, even qualitatively. The pulsatile secretion of GnRH that generally stems from the pulse dynamics of neurons [10] is studied in $[26,14,18,11]$ and [3]. When the concentration of serum Te rises, the pulses of GnRH become sparser and their amplitude (or area) diminishes [24].

To capture the above described pulsatile feedback mechanism, the GnRH producing cells of hypothalamus can be modeled as a pulse element implementing pulseamplitude and pulse-frequency modulation [9]. Then Te is the modulating signal and $\mathrm{GnRH}$ is the modulated pulse signal. The pulsatile LH secretion can be seen as the response of the continuous part of the system on the pulse signaling of the hypothalamus. 


\subsection{Model formulation}

Consider now model (2), where $f(T)$ is no longer a nonlinear function, but rather an operator describing pulse-amplitude-frequency modulation. There are not so many kinds of pulse-amplitude modulation, they are mostly related to the choice of the pulse form, but there is a rich variety of pulse-frequency modulation schemes and some of them are described in [9]. Unfortunately, no currently available biological evidence can substantiate the choice of one of the latter. At the same time, given the low pulse rate in the considered system, the choice of a certain modulation scheme can have a major impact on the closed-loop dynamics.

Let us denote $x_{1}=R(t), x_{2}=L(t), x_{3}=T(t)$. Consider a system

$$
\begin{aligned}
& \dot{x}_{1}=-b_{1} x_{1}+\xi(t), \\
& \dot{x}_{2}=-b_{2} x_{2}+g_{1} x_{1}, \\
& \dot{x}_{3}=-b_{3} x_{3}+g_{2} x_{2},
\end{aligned}
$$

where $b_{1}, b_{2}, b_{3}, g_{1}, g_{2}$ are positive parameters,

$$
\xi(t)=\sum_{n=0}^{\infty} \lambda_{n} \delta\left(t-t_{n}\right)
$$

Here $\delta(t)$ is the Dirac delta-function. Suppose that the GnRH pulse firing times $t_{n}$ are given by

$$
t_{n+1}=t_{n}+\tau_{n}, \quad \tau_{n}=\Phi\left(x_{3}\left(t_{n}\right)\right),
$$

where $\Phi(\cdot)$ is a frequency modulation characteristic, and

$$
\lambda_{n}=F\left(x_{3}\left(t_{n}\right)\right),
$$

where $F(T)$ is an amplitude modulation characteristic. Without loss of generality, assume $t_{0}=0$. Unlike modulators used in technical applications, $\Phi(\cdot)$ is nondecreasing and $F(\cdot)$ is non-increasing. The functions $\Phi$ and $F$ are positive and have the constant bounds due to physiological limitations

$$
0<\Phi_{1} \leqslant \Phi(\cdot) \leqslant \Phi_{2}, \quad 0<F_{1} \leqslant F(\cdot) \leqslant F_{2} .
$$

Thus (5), (6) denote a combined (pulse frequency and pulse amplitude) modulation of Type 1, [9].

The first equation in (3) can be equivalently rewritten as a differential equation

$$
\dot{x}_{1}=-b_{1} x_{1}, \quad t_{n}<t<t_{n+1},
$$

with jumps

$$
x_{1}\left(t_{n}+0\right)=x_{1}\left(t_{n}-0\right)+\lambda_{n} .
$$


Thus the static nonlinear functions $\Phi(\cdot)$ and $F(\cdot)$, together with the decay rate $b_{1}$, completely define the mechanism of the pulsatile feedback. Notice here that Dirac delta sequence (4) is an auxiliary artifact and has no effect on the boundedness of the biologically meaningful variables in the model.

System (3) in vector-matrix form with $x \in R^{3}$ is

$$
\frac{d x}{d t}=A x+B \xi(t), \quad y=C x,
$$

where

$$
A=\left[\begin{array}{ccc}
-b_{1} & 0 & 0 \\
g_{1} & -b_{2} & 0 \\
0 & g_{2} & -b_{3}
\end{array}\right], \quad B=\left[\begin{array}{l}
1 \\
0 \\
0
\end{array}\right], \quad C^{T}=\left[\begin{array}{l}
0 \\
0 \\
1
\end{array}\right] .
$$

The jump conditions are

$$
x\left(t_{n}+0\right)=x\left(t_{n}-0\right)+\lambda_{n} B .
$$

Obviously, $A$ is Hurwitz stable and $C B=0$. Equation (7) does not have equilibria because all the modulation characteristics are positive.

\subsection{Boundedness of solutions}

A plausible mathematical model of hormone regulation should not produce unbounded solutions. The result below proves that the solutions of (3)-(6) are always bounded from below and above.

Theorem 1 All the solutions of system (3)-(6) stay within the following limits

$$
V_{i} \leqslant \liminf _{t \rightarrow+\infty} x_{i}(t) \leqslant \limsup _{t \rightarrow+\infty} x_{i}(t) \leqslant H_{i}, \quad i=1,2,3
$$

where

$$
\begin{aligned}
V_{1} & =\frac{F_{1}}{e^{b_{1} \Phi_{2}}-1}, & H_{1} & =\frac{F_{2}}{1-e^{-b_{1} \Phi_{1}}}, \\
V_{2} & =\frac{g_{1} V_{1}}{b_{2}}, & H_{2} & =\frac{g_{1} H_{1}}{b_{2}}, \\
V_{3} & =\frac{g_{1} g_{2} V_{1}}{b_{2} b_{3}}, & H_{3} & =\frac{g_{1} g_{2} H_{1}}{b_{2} b_{3}} .
\end{aligned}
$$

Proof: see Appendix A. 


\section{Periodic solutions}

Since the processes of endocrine regulation are self-sustained, only periodic solutions of system (3)-(6) are treated here. Consider the translation operator along the trajectories $x(t)$ of (3)-(6):

$$
Q: x\left(t_{n}-0\right) \mapsto x\left(t_{n+1}-0\right) .
$$

Obviously,

$$
Q(x)=e^{A \Phi(C x)}(x+F(C x) B) .
$$

Following [27], a periodic solution is called $m$-cycle if there are exactly $m$ impulses of sequence (4) fired on its least period.

Then 1-cycle corresponds to a fixed point $x^{0}$ of the operator $Q(\cdot)$, i.e.

$$
Q\left(x^{0}\right)=x^{0} .
$$

and has the initial condition $x\left(t_{0}-0\right)=x^{0}$. The periodic solution corresponding to this mode is characterized by the period $\tau_{0}$ and the pulse amplitude $\lambda_{0}$. Denote $y^{0}=C x^{0}$.

Assume that the numbers $b_{1}, b_{2}, b_{3}$ are distinct. This assumption is biologically feasible since all the involved hormones have different half-life times. Introduce the numbers

$$
\begin{aligned}
\alpha_{1} & =\frac{1}{\left(b_{2}-b_{1}\right)\left(b_{3}-b_{1}\right)}, \\
\alpha_{2} & =\frac{1}{\left(b_{1}-b_{2}\right)\left(b_{3}-b_{2}\right)}, \\
\alpha_{3} & =\frac{1}{\left(b_{1}-b_{3}\right)\left(b_{2}-b_{3}\right)} .
\end{aligned}
$$

Obviously $\alpha_{1}+\alpha_{2}+\alpha_{3}=0$ and two of these numbers are positive, while the third number is negative.

Theorem 2 System (3)-(6) has one and only one 1-cycle. The cycle parameters $\lambda_{0}, \tau_{0}$ and $y^{0}$ can be evaluated by solving the following system of transcendental equations

$$
\begin{aligned}
& y^{0}=\lambda_{0} g_{1} g_{2} \sum_{i=1}^{3} \frac{\alpha_{i}}{e^{b_{i} \tau_{0}}-1}, \\
& \lambda_{0}=F\left(y^{0}\right), \quad \tau_{0}=\Phi\left(y^{0}\right) .
\end{aligned}
$$

Proof: see Appendix B. 
Notice that Theorem 2 says nothing about stability of the solution in question. From Theorem 1 it is known that all the solutions of model (3)-(6) are bounded but bifurcation is yet possible. In the sequel, stability is understood as orbital asymptotic stability with respect to small perturbations in the initial conditions.

Consider a periodic solution $x^{p}(t)$ of (3)-(6) with the initial condition $x^{p}\left(t_{0}-0\right)=$ $x^{0}$. Let $\Omega \subset \mathbb{R}^{3}$ be the trajectory corresponding to $x^{p}(t)$, i.e. the set of all points in the state space that the solution goes through

$$
\Omega=\bigcup_{\theta \in\left[t_{0}-0, \infty\right)}\left\{x^{p}(\theta)\right\}
$$

The solution $x^{p}(t)$ will be called stable, if there is a neighborhood $\mathcal{D}$ of $x_{0}$ such that for each solution $x(t)$ originating from $\mathcal{D}$ at $t_{0}-0$ the limit relationship

$$
\inf _{\xi \in \Omega}\|x(t)-\xi\| \rightarrow 0 \quad \text { as } \quad t \rightarrow \infty
$$

is valid for an arbitrary vector norm $\|\cdot\|$. Notice that the starting time is essential here. This type of stability is usually termed as orbital stability and does not generally imply stability in Lyapunov sense, see [20],[12].

Local stability of a 1-cycle can be checked by linearizing the mapping $Q(x)$ in a neighborhood of the fixed point $x^{0}$.

Theorem 3 Suppose that $x^{0}$ satisfies (12) and the functions $F(\cdot)$ and $\Phi(\cdot)$ have continuous derivatives $F^{\prime}(\cdot)$ and $\Phi^{\prime}(\cdot)$ in a neighborhood of $y^{0}=C x^{0}$. Then the 1 -cycle with the inital condition $x\left(t_{0}-0\right)=x^{0}$ is stable if

$$
A_{1}=e^{A \Phi\left(y^{0}\right)}\left[I+F^{\prime}\left(y^{0}\right) B C\right]+\Phi^{\prime}\left(y^{0}\right) A x^{0} C
$$

is Schur stable (i.e. all its eigenvalues lie strictly inside the unit circle).

Proof: see Appendix C.

For a 2-cycle, the initial conditions $x\left(t_{0}-0\right)=x^{0}$ solve the equation

$$
Q\left(Q\left(x^{0}\right)\right)=x^{0}
$$

Consider a 2-cycle $x^{p}(t)$ with the pulse parameters $\tau_{0}, \lambda_{0}, \tau_{1}, \lambda_{1}$. Denote

$$
\hat{x}^{0}=Q\left(x^{0}\right), \quad y^{0}=C x^{0}, \quad \hat{y}^{0}=C \hat{x}^{0} .
$$

Theorem 4 Suppose that $x^{0}$ satisfies (15). Then parameters of the 2-cycle with the 
initial value $x\left(t_{0}-0\right)=x^{0}$ satisfy the following transcendental equations

$$
\begin{array}{rlrl}
y^{0} & =g_{1} g_{2} \sum_{i=1}^{3} \alpha_{i} \frac{\lambda_{0}+\lambda_{1} e^{b_{i} \tau_{0}}}{e^{b_{i}\left(\tau_{0}+\tau_{1}\right)}-1}, \\
\hat{y}^{0} & =g_{1} g_{2} \sum_{i=1}^{3} \alpha_{i} \frac{\lambda_{1}+\lambda_{0} e^{b_{i} \tau_{1}}}{e^{b_{i}\left(\tau_{0}+\tau_{1}\right)}-1}, \\
\lambda_{0} & =F\left(y^{0}\right), & \tau_{0}=\Phi\left(y^{0}\right), \\
\lambda_{1} & =F\left(\hat{y}^{0}\right), & \tau_{1}=\Phi\left(\hat{y}^{0}\right) .
\end{array}
$$

Proof: see Appendix D.

Theorem 5 Let $F(\cdot)$ and $\Phi(\cdot)$ have continuous derivatives in some neighborhoods of $y^{0}$ and $\hat{y}^{0}$. Consider the matrix

$$
A_{2}=\hat{A}_{1} \hat{A}_{2}
$$

where

$$
\begin{aligned}
& \hat{A}_{1}=e^{A \Phi\left(\hat{y}^{0}\right)}\left[I+F^{\prime}\left(\hat{y}^{0}\right) B C\right]+\Phi^{\prime}\left(\hat{y}^{0}\right) A x^{0} C, \\
& \hat{A}_{2}=e^{A \Phi\left(y^{0}\right)}\left[I+F^{\prime}\left(y^{0}\right) B C\right]+\Phi^{\prime}\left(y^{0}\right) A \hat{x}^{0} C .
\end{aligned}
$$

Then the 2-cycle with the initial value $x\left(t_{0}-0\right)=x^{0}$ is stable if $A_{2}$ is Schur stable.

Proof: see Appendix E.

\section{Piecewise affine modulation functions}

Following [18], consider a special case when $F(\cdot)$ and $\Phi(\cdot)$ are piecewise affine. Namely, let

$$
\begin{gathered}
F(y)= \begin{cases}F_{2}, & 0 \leqslant y<\Delta_{1}, \\
-k_{F} y+b_{F}, & \Delta_{1} \leqslant y \leqslant \Delta_{2}, \\
F_{1}, & y>\Delta_{2},\end{cases} \\
\Phi(y)= \begin{cases}\Phi_{1}, & 0 \leqslant y<\Delta_{1}, \\
k_{\Phi} y+b_{\Phi}, & \Delta_{1} \leqslant y \leqslant \Delta_{2}, \\
\Phi_{2}, & y>\Delta_{2} .\end{cases}
\end{gathered}
$$

where

$$
0<F_{1}<F_{2}, \quad 0<\Phi_{1}<\Phi_{2}, \quad 0<\Delta_{1}<\Delta_{2}
$$


and $k_{F}, b_{F}, k_{\Phi}$ and $b_{\Phi}$ are selected to render continuous functions:

$$
\begin{array}{ll}
k_{F}=\frac{F_{2}-F_{1}}{\Delta_{2}-\Delta_{1}}, & b_{F}=\frac{\Delta_{2} F_{2}-\Delta_{1} F_{1}}{\Delta_{2}-\Delta_{1}}, \\
k_{\Phi}=\frac{\Phi_{2}-\Phi_{1}}{\Delta_{2}-\Delta_{1}}, & b_{\Phi}=\frac{\Delta_{2} \Phi_{1}-\Delta_{1} \Phi_{2}}{\Delta_{2}-\Delta_{1}} .
\end{array}
$$

Thus, the function $\Phi(y)$ is rising from $\Phi_{1}$ to $\Phi_{2}$, while $F(y)$ is falling from $F_{2}$ to $F_{1}$. The functions saturate simultaneously and are constant on two intervals $y \leqslant \Delta_{1}$ and $y \geqslant \Delta_{2}$.

Consider a solution $x(t)$ of (3)-(6). The solution $x(t)$ will be called saturated, if all the values

$$
y_{n}=C x\left(t_{n}-0\right), \quad n=0,1, \ldots,
$$

belong to the saturation intervals of $F(y)$ and $\Phi(y)$. The solution $x(t)$ will be called semi-saturated, if some of the values (18) belong to the saturation intervals, and some do not.

Theorem 6 Any saturated m-cycle is stable.

Proof: Indeed, apply Theorems 3, 5 with $F^{\prime}=0, \Phi^{\prime}=0$. Then

$$
A_{1}=e^{A \Phi\left(y^{0}\right)}, \quad A_{2}=e^{A \Phi\left(\hat{y}^{0}\right)} e^{A \Phi\left(y^{0}\right)} .
$$

Since the matrix $A$ is triangular, the eigenvalues of $A_{1}$ are $e^{-b_{i} \Phi\left(y^{0}\right)}$ and the eigenvalues of $A_{2}$ are $e^{-b_{i} \Phi\left(\hat{y}^{0}\right)} e^{-b_{i} \Phi\left(y^{0}\right)}$. Obviously, these numbers are less than one. By applying similar reasoning to any solution with $m>2$, it is concluded that such a solution also is stable.

The piecewise affine character of $\varphi$ enables a systematic analysis of saturated periodic solutions of model (7). Direct calculations yield the following saturation conditions.

Proposition 1 A saturated 1-cycle exists iff one of the following inequalities holds:

$$
\begin{aligned}
& g_{1} g_{2} F_{2} \sum_{i=1}^{3} \frac{\alpha_{i}}{e^{b_{i} \Phi_{1}}-1} \leqslant \Delta_{1}, \\
& g_{1} g_{2} F_{1} \sum_{i=1}^{3} \frac{\alpha_{i}}{e^{b_{i} \Phi_{2}}-1} \geqslant \Delta_{2} .
\end{aligned}
$$

Proposition 2 A saturated 2-cycle exists iff one of the following inequalities is 
satisfied:

$$
\begin{gathered}
g_{1} g_{2} F_{2} \sum_{i=1}^{3} \alpha_{i} \frac{e^{b_{i} \Phi_{1}}+1}{e^{2 b_{i} \Phi_{1}}-1} \leqslant \Delta_{1} ; \\
g_{1} g_{2} F_{1} \sum_{i=1}^{3} \alpha_{i} \frac{e^{b_{i} \Phi_{2}}+1}{e^{2 b_{i} \Phi_{2}}-1} \geqslant \Delta_{2} ; \\
\min \left\{y^{0}, \hat{y}^{0}\right\} \leqslant \Delta_{1}, \quad \max \left\{y^{0}, \hat{y}^{0}\right\} \geqslant \Delta_{2},
\end{gathered}
$$

where

$$
\begin{aligned}
& y^{0}=g_{1} g_{2} \sum_{i=1}^{3} \alpha_{i} \frac{F_{2}+F_{1} e^{b_{i} \Phi_{1}}}{e^{b_{i}\left(\Phi_{1}+\Phi_{2}\right)}-1} \\
& \hat{y}^{0}=g_{1} g_{2} \sum_{i=1}^{3} \alpha_{i} \frac{F_{1}+F_{2} e^{b_{i} \Phi_{2}}}{e^{b_{i}\left(\Phi_{1}+\Phi_{2}\right)}-1} .
\end{aligned}
$$

\section{Simulation results}

The values of the model parameters in this section are not biologically motivated but rather chosen to clearly illustrate the dynamical behaviors of the system.

Consider piecewise affine $F(\cdot)$ and $\Phi(\cdot)$ with

$$
\Delta_{1}=1.5, \Delta_{2}=4, \Phi_{1}=60, \Phi_{2}=100, F_{1}=3, F_{2}=5 .
$$

In simulation, the following periodic solutions of system (3)-(6) have been observed

- The system exhibits a stable 1-cycle.

- The system exhibits a stable 2-cycle. Besides, there is an unstable 1-cycle.

No other types of behavior are found.

As an example, consider different types of behavior in system (7) arising from variation of the element $b_{1}$ in the matrix of the linear part

$$
A=\left[\begin{array}{ccc}
-b_{1} & 0 & 0 \\
2 & -0.15 & 0 \\
0 & 0.5 & -0.2
\end{array}\right] \text {. }
$$

This corresponds to alternations in the clearing rate of $\mathrm{GnRH}$.

(1) For $b_{1} \leqslant 0.03$ the system has a stable saturated 1-cycle $\left(\lambda_{n}=3\right)$. 

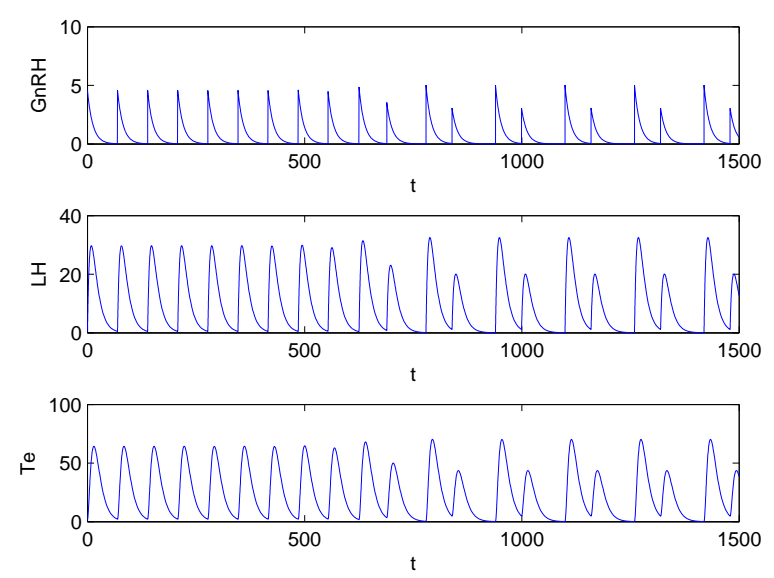

Fig. 1. Periodic solution for $b_{1}=0.08$. 1-cycle evolves to 2-cycle.

(2) For $b_{1}=0.04$ the system has an unstable unsaturated 1-cycle and a stable semi-saturated 2-cycle (saturation with $\lambda_{n}=3$ ).

(3) For $0.05 \leqslant b_{1} \leqslant 0.08$, the system has an unstable unsaturated 1-cycle and a stable saturated 2-cycle.

(4) $0.09 \leqslant b_{1} \leqslant 0.11$ the system has an unstable unsaturated 1 -cycle and a stable semi-saturated 2-cycle (saturation with $\lambda_{n}=5$ ).

(5) For $b_{1} \geqslant 0.12$ the system has a stable saturated 1-cycle $\left(\lambda_{n}=5\right)$.

The intervals $(0.03,0.04)$ and $(0.11,0.12)$ contain the values of $b_{1}$, for which a period-doubling bifurcation takes place, [27]. It worth to notice that classical bifurcation theory does not apply in this case since the functions $F^{\prime}(\cdot)$ and $\Phi^{\prime}(\cdot)$ are not continuous.

The graphs of the transition from the unstable mode with single pulse on the period to the stable mode with a double pulse on the period are shown in Fig. 1. Periodic solutions in the state space are depicted in Fig. 2. Notice the prominent discontinuous behavior of the model along GnRH axis, due to the pulse-modulated feedback.

\section{Partial model validation on experimental data}

In the previous section, it is shown that the model under consideration is capable of sustained nonlinear oscillations with two pulses of $\mathrm{GnRH}$ on the least period. In medical literature, it is an established thesis that pulses of GnRH from hypothalamus cause pulses of LH secretion in pituitary in a nearly uniformly one-to-one ratio, [23]. In a typical endocrinological study, pulses of GnRH and LH are counted using a pulse detection algorithm run on a hormone concentration time series, as in the classical CLUSTER algorithm by Veldhuis and Johnson, [25]. Pulses are recognized over a sliding window as significant increases followed by significant 


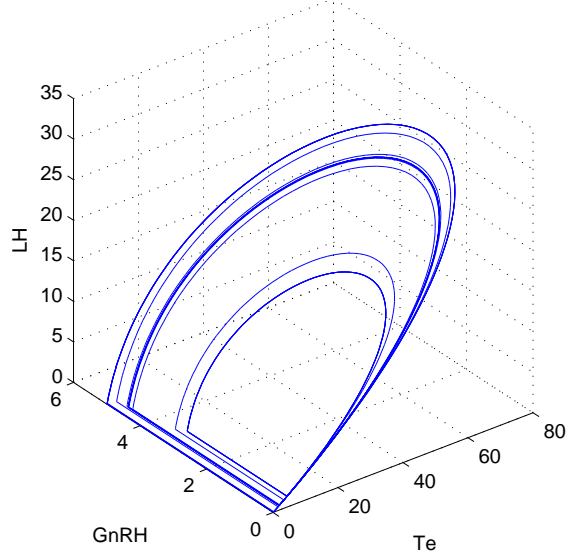

Fig. 2. Trajectories in the state space. Notice the discontinuous behavior along GnRH axis due to pulse modulated feedback.

decreases. In this way, minor pulses can be missed due to slow sampling or regarded as nuisance. In this section, it is demonstrated that the signal form predicted by the model studied in this paper for the case of two pulses of GnRH on the least period is consistent with LH serum concentration data observed in a young human male at a sampling time of $10 \mathrm{~min}$. The data set was kindly provided by Prof. Veldhuis of Mayo Clinic.

In the model, the amplitude and onset time of $\mathrm{GnRH}$ pulses are governed by weighted $\delta$-functions generated by the impulse feedback controller, see Fig. 3. The spiky pulses of GnRH cause much more sluggish pulses of LH. Assuming that the periodic mode has two pulses of $\mathrm{GnRH}$ at the least period $T$ and setting $t=0$ at the beginning of a period, gives two weighted $\delta$-functions produced by the impulse controller in the mathematical model on each period

$$
\Theta(t)=\lambda_{0} \delta(t)+\lambda_{1} \delta\left(t-t_{1}\right)
$$

This results in the following evolution in the concentration of $\mathrm{GnRH}$

$$
\begin{array}{lrl}
R(t) & =\lambda_{0} e^{-b_{1} t}, & 0 \leqslant t<t_{1}, \\
R(t) & =\eta\left(b_{1}\right) e^{-b_{1} t}, \eta(x)=\lambda_{0}+\lambda_{1} e^{x t_{1}}, & t_{1} \leqslant t<T,
\end{array}
$$

and of the measured model output LH

$$
\begin{array}{ll}
L(t)=\frac{\lambda_{0} g_{1}}{b_{2}-b_{1}}\left(e^{-b_{1} t}-e^{-b_{2} t}\right), & 0 \leqslant t<t_{1}, \\
L(t)=\frac{g_{1}}{b_{2}-b_{1}}\left(\eta\left(b_{1}\right) e^{-b_{1} t}-\eta\left(b_{2}\right) e^{-b_{2} t}\right), & t_{1} \leqslant t<T .
\end{array}
$$

Since GnRH concentrations cannot be measured in the human, the problem of evaluating the model parameters $b_{1}, b_{2}, g_{1}, \lambda_{0}, \lambda_{1}$ from experimental data involves deconvolution. From the equations above, it becomes clear that $\lambda_{0}$ and $g_{1}$ cannot be 
estimated separately from measurements of LH but only in a product. Therefore, the values of $\lambda_{0}$ and $\lambda_{1}$ cannot neither be estimated but only their ratio.

According to [26],

$$
\begin{gathered}
0.23 \mathrm{~min}^{-1} \leqslant b_{1} \leqslant 0.69 \mathrm{~min}^{-1}, \\
0.0087 \mathrm{~min}^{-1} \leqslant b_{2} \leqslant 0.014 \mathrm{~min}^{-1} .
\end{gathered}
$$

The rise time of an LH pulse, defined as the time interval between the onset of the pulse and the time when the hormone concentration achieves its highest value, is independent of the driving $\delta$-function sequence and is expected to be within the following bounds

$$
5.75 \min \leqslant t_{\max }=\frac{\ln b_{1}-\ln b_{2}}{b_{1}-b_{2}} \leqslant 14.75 \min .
$$

A general recommendation for the choice of sampling time is to have 3-10 samples on the rise time interval. With the sampling time of $10 \mathrm{~min}$ in the data set, the system is definitely undersampled.

Using standard nonlinear least squares, the model parameters have been estimated from a data set representing a LH pulse, see Fig. 4. The following parameter values have been obtained $b_{1}=0.07, b_{2}=0.033, t_{1}=89, \lambda_{1}=0.04$. Assuming $\lambda_{0}=1.0$, the feedforward gain from GnRH to LH is estimated to $g_{1}=0.23$. Despite the reasonably good agreement between the identified model and the underlying data, the estimated parameters are far outside of the intervals indicated in medical literature, cf (19). The estimated elimination rate of $\mathrm{GnRH}$ is much slower than expected while the estimated elimination rate of $\mathrm{LH}$ is twice that of the highest expected one. On the one hand, it is plausible to assume that considerable differences exist between the biochemical reactions in vitro and in vivo. Besides, the parameter estimates are inaccurate due to the slow blood analysis sampling.

Notably, the second impulse of GnRH is quite small and amounts to only $4 \%$ of the first one. However, its impact on the signal form is well pronounced and repeats itself throughout the whole data set recorded on this particular patient. Data for other patients do not necessarily follow the same dynamic pattern. The observed behavior is apparently not an artifact since its is not related to certain regular assay timing or hormone concentration level.

\section{Conclusions}

The process of pulsatile endocrine regulation can suitably be described by means of pulse-modulated systems. A simple dynamic model of GnRH-LH-Te axis in the 

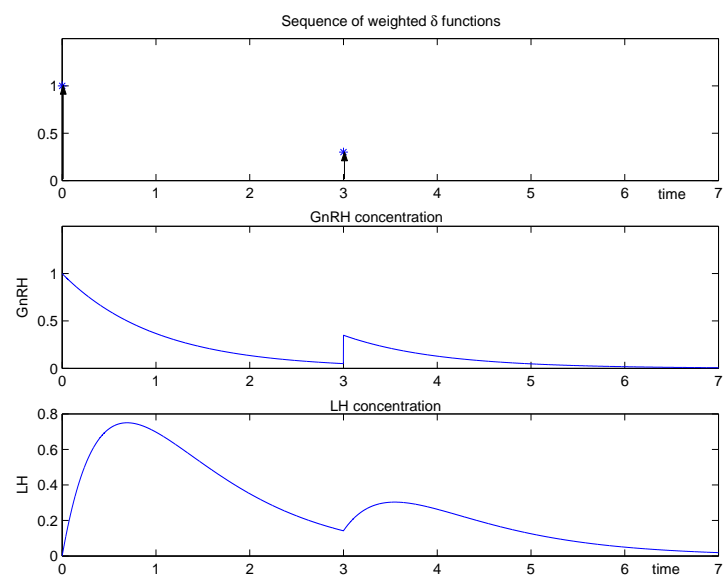

Fig. 3. Pulse of LH corresponding to two pulses of GnRH. Model parameters and the time scale are not biologically motivated.

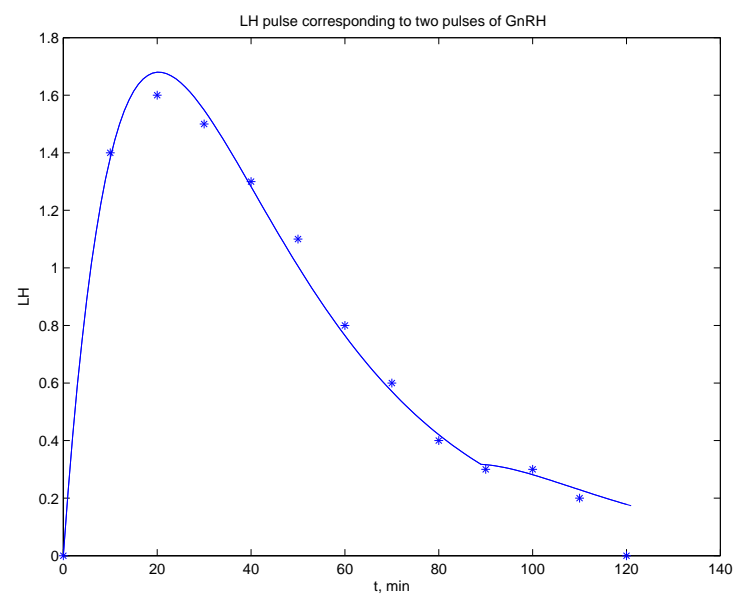

Fig. 4. Model with identified parameters (solid line) and two estimated pulses of GnRH compared to experimental data $(*$ denote measurements).

male is suggested and shown to produce stable periodic solutions with one or two GnRH pulses on the least period. Results on existence, uniqueness and stability of these periodic solutions are provided. The periodic mode with two GnRH pulses on the least period has not yet been described in medical literature but is supported by experimental data.

In clinical or experimental data, the regularity of hormone oscillations is heavily perturbed by many impacting factors. Both the amplitudes and periods of $\mathrm{GnRH}$ pulses are subject to significant variations which are often described as stochastic. However, the underlying dynamics of the GnRH-LH-Te axis can probably be explained by simple deterministic models, provided they take into account the feedback mechanism of hormonal regulation. 


\section{Acknowledgements}

This work has been carried out with financial support by The Royal Swedish Academy of Sciences. A. N. Churilov was supported in part by the Russian Foundation for Basic Research (project 05-01-00290-a). A. Medvedev was partly supported by Swedish Research Council.

The authors would like to express their gratitude to Prof. Johannes D. Veldhuis of Mayo Clinic for kindly providing clinical hormone data and encouraging this project.

\section{References}

[1] M. Cartwright and M. Husain. A model for the control of testosterone secretion. $J$. Theor. Biol., 123(2):239-250, 1986.

[2] A.N. Churilov, A.V. Medvedev, and A.I. Shepeljavyi. Mathematical models of testosterone regulation. In Stochastic Optimization in Informatics, (In Russian), number 2, pages 147-158. St. Petersburg State University, 2006.

[3] F. Clément and J.-P. Françoise. Mathematical modeling of the GnRH-pulse and surge generator. Online publication, http://fr.arxiv.org/pdf/q-bio.TO/0511023, 2005.

[4] D.J. Dierschke, A.N. Bhattacharya, L.E. Atkinson, and E. Knobil. Circhoral oscillations of plasma lh levels in the ovariectomized rhesus monkey. Endocrinology, 87:850-853, 1970.

[5] D.V. Efimov. Robust and adaptive control of nonlinear oscillations, (In Russian). Nauka, St. Petersburg, 2005.

[6] G. Enciso and E.D. Sontag. On the stability of a model of testosterone dynamics. $J$. Math. Biol., 49:627-634, 2004.

[7] L.S. Farhy. Modeling of oscillations of endocrine networks with feedback. Methods in Enzymology, 384:54-81, 2004.

[8] A.Kh. Gelig. Dynamics of nonlinear impulsive systems and neural nets, (In Russian). Leningrad Univ. Press., Leningrad, 1982.

[9] A.Kh. Gelig and A.N. Churilov. Stability and oscillations of nonlinear pulsemodulated systems. Birkhäuser, Boston, 1998.

[10] W. Gerstner and W.M. Kistler. Spiking neuron models: single neurons, populations, plasticity. Cambridge Univ. Press., Cambridge, 2002.

[11] F. Van Goor, A.P. LeBeau, L.Z. Krsmanovic, A. Sherman, K.J. Catt, and S.S. Stojilkovic. Amplitude-dependent spike-broadening and enhanced $\mathrm{Ca}^{2+}$ signaling in GnRH-secreting neurons. Biophys. J., 79:1310-1323, 2000. 
[12] J.K. Hale and H. Koçak. Dynamics and bifurcations. Springer, New York, 1991.

[13] W.J. Heuett and H. Qian. A stochastic model of oscillatory blood testosterone levels. Bull. Math. Biol., 68(6):1383-1399, 2006.

[14] D.M. Keenan, W. Sun, and J.D. Veldhuis. A stochastic biomathematical model of male reproductive hormone systems. SIAM J. Appl. Math., 61(3):934-965, 2000.

[15] L.Z. Krsmanović, S.S. Stojilković, F. Merelli, S.M. Dufour, M.A. Virmani, and K.J. Catt. Calcium signaling and episodic secretion of gonadotropin-releasing hormone in hypothalamic neurons. Proc. Nat. Acad. Sci. USA, 89:8462-8466, 1992.

[16] V.D. Milman and A.D. Myshkis. On stability of motion with pushes. Siberian Math. J., 1(2):233-267, 1960. (In Russian).

[17] J.D. Murray. Mathematical biology, I: An introduction (3rd ed.). Springer, New York, 2002.

[18] N.L. Rasgon, L. Pumphrey, P. Prolo, S. Elman, A.B. Negrao, J. Licinio, and A. Garfinkel. Emergent oscillations in mathematical model of the human menstrual cycle. CNS Spectrums, 8(11):805-814, 2003.

[19] E.N. Rosenwasser. Periodically nonstationary control systems, (In Russian). Nauka, Moscow, 1973.

[20] P.E. Simeonov and D.D. Bainov. Orbital stability of periodic solutions of autonomous systems with impulse effect. Internat. J. Systems Sci., 19:2562-2585, 1988.

[21] R.W. Smith. Hypothalamic regulation of pituitary secretion of luteinizing hormone II. feedback control of gonadotropin secretion. Bull. Math. Biol., 42(1):57-78, 1980.

[22] R.W. Smith. Qualitative mathematical models of endocrine systems. Amer. J. Physiol., 245(4):R473-R477, 1983.

[23] J.D. Veldhuis. The hypotalamic-pituitary-testicular axis. In S. S. C. Yen and R. B. Jaffe, editors, Reproductive Endocrinology, (3rd ed.), pages 409-459. Saunders, Philadelphia, PA, 1991.

[24] J.D. Veldhuis. Recent insights into neuroendocrine mechanisms of aging of the human male hypothalamic-pituitary-gonadal axis. J. Andrology, 20(1):1-18, 1999.

[25] J.D. Veldhuis and M.L. Johnson. Cluster analysis: a simple, versatile, and robust algorithm for endocrine pulse detection. Am. J. Physiol. Endocrinol. Metab., 250:E486-E493, 1986.

[26] J.D. Veldhuis and D.M. Keenan. A biomathematical model of time-delayed feedback in the human male hypothalamic-pituitary-Leydig cell axis. Am. J. Physiol., 275 (Endocrinol. Metab. 38):E157-E176, 1998.

[27] Zh.T. Zhusubaliev and E. Mosekilde. Bifurcations and chaos in piecewise-smooth dynamical systems. World Scientific, Singapore, 2003. 


\section{A Proof of Theorem 1}

The proof generally follows the method of $[16,8]$. Let $t_{n} \leqslant t<t_{n+1}$. Then

$$
\begin{aligned}
& x_{1}(t)=e^{-b_{1}\left(t-t_{n}\right)} x_{1}\left(t_{0}-0\right)+\tilde{x}_{1}(t), \\
& \tilde{x}_{1}(t)=\sum_{k=0}^{n} \lambda_{k} e^{-b_{1}\left(t-t_{k}\right)} .
\end{aligned}
$$

The first term vanishes as $t \rightarrow \infty$, so it suffices to establish bounds on $\tilde{x}_{1}(t)$. Since $F_{1} \leqslant \lambda_{n} \leqslant F_{2}$ and $0 \leqslant t-t_{n} \leqslant \Phi_{2}$,

$$
F_{1} e^{-b_{1} \Phi_{2}} \sum_{k=0}^{n} e^{-b_{1}\left(t_{n}-t_{k}\right)} \leqslant \tilde{x}_{1}(t) \leqslant F_{2} \sum_{k=0}^{n} e^{-b_{1}\left(t_{n}-t_{k}\right)} .
$$

Using the equality

$$
t_{n}-t_{k}=\left(t_{n}-t_{n-1}\right)+\left(t_{n-1}-t_{n-2}\right)+\ldots+\left(t_{k+1}-t_{k}\right),
$$

it follows

$$
(n-k) \Phi_{1} \leqslant t_{n}-t_{k} \leqslant(n-k) \Phi_{2} .
$$

Now, (9) can be obtained by summing two geometric series. Bounds (10) and (11) follow from (9) and (3), respectively.

\section{B Proof of Theorem 2}

Let us prove that a solution $x^{0}$ of (12) exists and is unique. Equation (12) is equivalent to

$$
x^{0}=F\left(y^{0}\right)\left(e^{-A \Phi\left(y^{0}\right)}-I\right)^{-1} B .
$$

with $y^{0}=C x^{0}$. Then (B.1) implies

$$
y^{0}=F\left(y^{0}\right) C\left(e^{-A \Phi\left(y^{0}\right)}-I\right)^{-1} B .
$$

The converse statement is also true: if $y^{0}$ satisfies (B.2), then (B.1) gives a solution of (12). (Equation (B.2) is sometimes called "equation of periods" [19,9].) By assumption, the function $F(y)$ is positive and non-increasing. Consider a scalar function

$$
U(y)=C\left(e^{-A \Phi(y)}-I\right)^{-1} B .
$$

To prove the theorem it suffices to show that $U(y)$ is also positive and non-increasing. Since $\Phi(y)$ is positive and non-decreasing, we only need to prove that the function

$$
U_{0}(y)=C\left(e^{-A y}-I\right)^{-1} B
$$


is positive and non-increasing for $y>0$. Because $A$ is Hurwitz stable, $U_{0}(y)$ vanishes as $y \rightarrow \infty$. Let us show that the derivative of $U_{0}(y)$ is non-positive. Direct evaluation of the matrix exponential gives

$$
e^{A t}=\left[\begin{array}{ccc}
e^{-b_{1} t} & 0 & 0 \\
E_{21}(t) & e^{-b_{2} t} & 0 \\
E_{31}(t) & E_{32}(t) & e^{-b_{3} t}
\end{array}\right],
$$

where

$$
\begin{aligned}
& E_{21}=\frac{g_{1}}{b_{2}-b_{1}}\left(e^{-b_{1} t}-e^{-b_{2} t}\right), \\
& E_{32}=\frac{g_{2}}{b_{3}-b_{2}}\left(e^{-b_{2} t}-e^{-b_{3} t}\right), \\
& E_{31}=g_{1} g_{2} \sum_{i=1}^{3} \alpha_{i} e^{-b_{i} t} .
\end{aligned}
$$

By streightforward computations it can be shown that

$$
U_{0}(y)=g_{1} g_{2} \sum_{i=1}^{3} \frac{\alpha_{i}}{e^{b_{i} y}-1}
$$

With the help of this representation (B.2) can be rewritten in the form (13). It follows that

$$
U_{0}^{\prime}(y)=g_{1} g_{2} \sum_{i=1}^{3}\left(-\alpha_{i}\right) \phi_{y}\left(b_{i}\right), \quad \phi_{y}(t)=\frac{t e^{t y}}{\left(e^{t y}-1\right)^{2}}
$$

Since $U_{0}$ is symmetric in $b_{i}$, suppose, without loss of generality, that $b_{3}>b_{2}>b_{1}$. Then $\alpha_{1}>0, \alpha_{3}>0$ and $\alpha_{2}<0$. The function $U_{0}(y)$ is non-increasing for $y>0$ if

$$
\alpha_{1} \phi_{y}\left(b_{1}\right)+\alpha_{3} \phi_{y}\left(b_{3}\right) \geqslant\left(-\alpha_{2}\right) \phi_{y}\left(b_{2}\right) .
$$

Define a number $\nu=-\alpha_{1} / \alpha_{2}$. Then $0<\nu<1$ and (B.3) can be written as

$$
\begin{gathered}
\nu \phi_{y}\left(b_{1}\right)+(1-\nu) \phi_{y}\left(b_{3}\right) \geqslant \phi_{y}\left(b_{2}\right), \\
b_{2}=\nu b_{1}+(1-\nu) b_{3} .
\end{gathered}
$$

It can be verified that $\phi_{y}^{\prime \prime}(t) \geqslant 0$ for $y>0, t>0$. So the function $\phi_{y}(t)$ is convex and (B.4) is satisfied.

\section{Proof of Theorem 3}

Denote $X_{n}=x\left(t_{n}-0\right)$. Then $X_{n}$ satisfies a discrete time equation

$$
X_{n+1}=Q\left(X_{n}\right)
$$


and $X_{n} \equiv x^{0}$ is an equilibrium of (C.1). Expanding $Q(x)$ in a series in a neighborhood of $x^{0}$ one obtains

$$
Q(x)=Q\left(x^{0}\right)+A_{1}\left(x-x^{0}\right)+o\left(\left\|x-x^{0}\right\|\right) \quad \text { as } \quad x \rightarrow x^{0},
$$

so the equilibrium $x^{0}$ is locally asymptotically stable since $A_{1}$ is Schur.

Denote

$$
a=F\left(C x^{0}\right), \quad \omega=\Phi\left(C x^{0}\right) .
$$

Let $x^{p}(t)$ be a 1-cycle starting from $x^{0}$ at $t=t_{0}-0$

$$
\begin{aligned}
x^{0} & =e^{A \omega}\left(x^{0}+a B\right), \\
x^{p}(t) & =e^{A t}\left(x^{0}+a B\right), \quad 0<t<\omega .
\end{aligned}
$$

Let us take a solution $x(t)$ starting at $t=t_{0}-0$ in a sufficiently small neighborhood of $x^{0}$. A it was shown above, $x\left(t_{n}-0\right) \rightarrow x^{0}$ as $n \rightarrow \infty$. Since the functions $F(\cdot)$ and $\Phi(\cdot)$ are continuous and

$$
t_{n+1}-t_{n}=\Phi\left(C x\left(t_{n}-0\right)\right), \quad \lambda_{n}=F\left(C x\left(t_{n}-0\right)\right),
$$

we conclude that

$$
t_{n+1}-t_{n} \rightarrow \omega, \quad \lambda_{n} \rightarrow a \quad \text { as } \quad n \rightarrow \infty
$$

Since

$$
x\left(t_{n}+0\right)=x\left(t_{n}-0\right)+\lambda_{n} B, \quad x^{p}\left(t_{0}+0\right)=x^{0}+a B,
$$

we get

$$
x\left(t_{n}+0\right) \rightarrow x^{p}\left(t_{0}+0\right) \quad \text { as } \quad n \rightarrow \infty .
$$

For any integer $n$ one has

$$
x(t)=e^{A\left(t-t_{n}\right)}\left(x\left(t_{n}+0\right)+\lambda_{n} B\right), \quad t_{n}<t<t_{n+1} .
$$

Rewrite this formula as

$$
\begin{aligned}
& x(t)=e^{A\left(t-t_{n}\right)}\left(x\left(t_{n}+0\right)-x^{0}-a B\right)+\tilde{x}(t), \\
& \tilde{x}(t)=e^{A\left(t-t_{n}\right)}\left(x^{0}+a B\right) .
\end{aligned}
$$

As we consider $t_{n}<t<t_{n+1}$, we have $0<t-t_{n}<\Phi_{2}$ and the value $\left\|e^{A\left(t-t_{n}\right)}\right\|$ is bounded uniformly in $n$ and $t$. In view of (C.4), we conclude that the first additive in (C.5) vanishes as $n \rightarrow \infty$.

Consider $\tilde{x}(t)$. Firstly, suppose that

$$
t_{n}<t<\min \left\{t_{n+1}, t_{n}+\omega\right\} .
$$

Then (C.3) and (C.6) yield $\tilde{x}(t)=x^{p}\left(t-t_{n}\right) \in \Omega$. 
Now suppose that $t_{n+1}>t_{n}+\omega$ and $t_{n}+\omega \leqslant t<t_{n+1}$. Since $e^{A\left(t-t_{n}\right)}=$ $e^{A\left(t-t_{n}-\omega\right)} e^{A \omega}$, one gets

$$
\tilde{x}(t)=e^{A\left(t-t_{n}-\omega\right)} x^{0}, \quad t_{n}+\omega \leqslant t<t_{n+1} .
$$

Because

$$
0 \leqslant t-t_{n}-\omega \leqslant t_{n+1}-t_{n}-\omega \rightarrow 0 \text { as } n \rightarrow \infty,
$$

$\tilde{x}(t)$ approaches $x^{0} \in \Omega$ as $n \rightarrow \infty$. The proof is complete.

\section{Proof of Theorem 4}

Equation (15) is equivalent to a system of transcendental equations

$$
\begin{aligned}
& x^{0}=\left[e^{-A\left(\tau_{0}+\tau_{1}\right)}-I\right]^{-1}\left(\lambda_{0} I+\lambda_{1} e^{-A \tau_{0}}\right) B, \\
& \hat{x}^{0}=\left[e^{-A\left(\tau_{0}+\tau_{1}\right)}-I\right]^{-1}\left(\lambda_{1} I+\lambda_{0} e^{-A \tau_{1}}\right) B, \\
& y^{0}=C x^{0}, \quad \lambda_{0}=F\left(y^{0}\right), \quad \tau_{0}=\Phi\left(y^{0}\right), \\
& \hat{y}^{0}=C \hat{x}^{0}, \quad \lambda_{1}=F\left(\hat{y}^{0}\right), \quad \tau_{1}=\Phi\left(\hat{y}^{0}\right) .
\end{aligned}
$$

By direct calculations it can be verified that

$$
\begin{aligned}
C\left[e^{-A\left(\tau_{0}+\tau_{1}\right)}-I\right]^{-1} e^{-A \tau} B & =g_{1} g_{2} \sum_{i=1}^{3} \alpha_{i} \frac{e^{b_{i} \tau}}{e^{b_{i}\left(\tau_{0}+\tau_{1}\right)}-1}
\end{aligned}
$$

for any number $\tau$. Excluding $x^{0}, \hat{x}^{0}$ from (D.1) we come to equations of periods in the form of (16).

\section{E Proof of Theorem 5}

Let $X_{n}=x\left(t_{n}-0\right)$. Then

$$
X_{n+2}=Q\left(Q\left(X_{n}\right)\right)
$$

In a small neigborhood of $x^{0}$ one obtains

$$
Q(Q(x))=Q\left(Q\left(x^{0}\right)\right)+A_{2}\left(x-x^{0}\right)+o\left(\left\|x-x^{0}\right\|\right)
$$

as $x \rightarrow x^{0}$. Then $X_{2 n} \rightarrow x^{0}$ as $n \rightarrow \infty$ provided that the initial perturbations are small enough. At the same time, $X_{2 n+1}=Q\left(X_{2 n}\right) \rightarrow \hat{x}^{0}=Q\left(x^{0}\right)$ as $n \rightarrow \infty$. 
The periodic solution $x^{p}(t)$ is characterized by the parameters

$$
\begin{array}{ll}
a=F\left(C x^{0}\right), & a_{1}=F\left(C \tilde{x}^{0}\right), \\
\omega=\Phi\left(C x^{0}\right), & \omega_{1}=\Phi\left(C x^{1}\right) .
\end{array}
$$

Then

$$
\begin{array}{ll}
x^{p}(t)=e^{A t}\left(x^{0}+a B\right), & 0<t<\omega, \\
x^{p}(t)=e^{A(t-\omega)}\left(\hat{x}^{0}+a_{1} B\right), & \omega<t<\omega_{1} .
\end{array}
$$

Applying the arguments given in the proof of Theorem 3 to the intervals $t_{2 n}<t<$ $t_{2 n+1}$ and $t_{2 n+1}<t<t_{2 n+2}$, leads to the stability statement. 